IZA DP No. 9015

Coming to Work While Sick: An Economic Theory of Presenteeism with an Application to German Data

Boris Hirsch

Daniel S. J. Lechmann

Claus Schnabel

April 2015 


\title{
Coming to Work While Sick: An Economic Theory of Presenteeism with an Application to German Data
}

\author{
Boris Hirsch \\ University of Erlangen-Nuremberg and IZA \\ Daniel S. J. Lechmann \\ University of Erlangen-Nuremberg \\ Claus Schnabel \\ University of Erlangen-Nuremberg and IZA
}

Discussion Paper No. 9015

April 2015

IZA
P.O. Box 7240
53072 Bonn
Germany

Phone: +49-228-3894-0

Fax: +49-228-3894-180

E-mail: iza@iza.org

\begin{abstract}
Any opinions expressed here are those of the author(s) and not those of IZA. Research published in this series may include views on policy, but the institute itself takes no institutional policy positions. The IZA research network is committed to the IZA Guiding Principles of Research Integrity.

The Institute for the Study of Labor (IZA) in Bonn is a local and virtual international research center and a place of communication between science, politics and business. IZA is an independent nonprofit organization supported by Deutsche Post Foundation. The center is associated with the University of Bonn and offers a stimulating research environment through its international network, workshops and conferences, data service, project support, research visits and doctoral program. IZA engages in (i) original and internationally competitive research in all fields of labor economics, (ii) development of policy concepts, and (iii) dissemination of research results and concepts to the interested public.
\end{abstract}

IZA Discussion Papers often represent preliminary work and are circulated to encourage discussion. Citation of such a paper should account for its provisional character. A revised version may be available directly from the author. 
IZA Discussion Paper No. 9015

April 2015

\section{ABSTRACT}

\section{Coming to Work While Sick: An Economic Theory of Presenteeism with an Application to German Data*}

Presenteeism, i.e. attending work while sick, is widespread and associated with significant costs. Still, economic analyses of this phenomenon are rare. In a theoretical model, we show that presenteeism arises due to differences between workers in (health-related) disutility from workplace attendance. As these differences are unobservable by employers, they set wages that incentivise sick workers to attend work. Using a large representative German data set, we test several hypotheses derived from our model. In line with our predictions, we find that bad health status and stressful working conditions are positively related to presenteeism. Better dismissal protection, captured by higher tenure, is associated with slightly fewer presenteeism days, whereas the role of productivity and skills is inconclusive.

JEL Classification: I19, J22

Keywords: presenteeism, absenteeism, sick leave, Germany

Corresponding author:

Claus Schnabel

University of Erlangen-Nuremberg

Chair of Labour and Regional Economics

Lange Gasse 20

90403 Nuremberg

Germany

E-mail: claus.schnabel@fau.de

\footnotetext{
* We would like to thank Daniel Arnold, Laszlo Goerke, and Harald Tauchmann for very useful suggestions. We further appreciate comments received from participants of the RES 2015 conference.
} 


\section{Motivation}

Why do employees come to work despite feeling sick (and being entitled to sick pay)? This phenomenon, which has been termed presenteeism (Aronsson et al. 2000$)$, is widespread in a large number of countries (see, e.g., Gosselin et al., 2013, for Canada, Vogt et al., 2009, Preisendörfer, 2010, and Oldenburg, 2012, for Germany, de Vroome, 2006, for the Netherlands, Hansen and Andersen, 2008, Böckerman and Laukkanen, 2009, and Johansen et al. 2014, for the Nordic countries, as well as Eurofound, 2012, and Arnold, 2015, for the EU). A survey by Eurofound (2012, p. 119) conducted in 2010 indicates that on average $39 \%$ of employees in EU countries attend work when being sick at least once a year, and in a number of countries this is even true for the majority of workers.

This behaviour is fascinating not alone in that it appears paradoxical at first sight, in particular when considering the rather generous sick pay in many western welfare states. Presenteeism is also important in that it goes along with several undesirable side effects. It is not just that sick workers are usually less productive $\oint^{1}$ going to work when ill may also spread infectious diseases to co-workers (see, e.g., Pichler and Ziebarth, 2015), exacerbate individuals' bad health status, and result in long-term health problems (as shown by Hansen and Andersen, 2009). Some observers (like Hemp, 2004) thus argue that presenteeism is even more costly and harmful than absenteeism, i.e. not showing up at work for whatever reason. Like absenteeism, presenteeism poses a challenge to firms devising organisational practices and wage policies that lead to an optimal amount of work attendance.

While there is a long and established literature on absenteeism (surveyed by Beemsterboer et al., 2009, and Treble and Barmby, 2011), presenteeism has received increased attention among researchers of different faculties as well as among practitioners and politicians only in recent years. So theoretical and empirical studies on the act of presenteeism are relatively rare (for surveys see Johns, 2010, and Steinke and Badura, 2011). The bulk of the literature is empirical and tries to identify in cross-sectional

1 Confusingly enough, the reduced productivity in case of presenteeism is also sometimes termed presenteeism in a strand of the literature that primarily focuses on the consequences of attending work while sick; see the reviews by Hemp (2004) and Schultz and Edington (2007). 
analyses the major health-related, individual, and organisational factors associated with the prevalence of presenteeism. A shortcoming of some of the extant studies is that they base inference about presenteeism on the examination of patterns of absence from work (see, e.g., Bierla et al., 2013), which is due to a lack of direct information on presenteeism. In recent years, more and more researchers have conducted or made use of surveys that include specific questions on presenteeism. Multivariate analyses for various countries indicate that presenteeism is related to health status and specific ailments, socio-demographic factors, work-related factors, employment conditions, and individuals' attitudes towards sickness absence (see, e.g., Aronsson and Gustafsson, 2005, Hansen and Andersen, 2008, Böckerman and Laukkanen, 2009, Gosselin et al., 2013, Pedersen and Skagen, 2014, Arnold, 2015, and the survey by Johns, 2010).

For Germany, on which we will use data in our empirical investigation, a factor analysis by Vogt et al. (2009) finds that presenteeism is lower for older employees and in firms with a better working atmosphere, whereas employees' limited control over work tasks and emotional stress as well as low job satisfaction are associated with higher presenteeism. In a logit estimation, Preisendörfer (2010) shows that fear of unemployment is associated with a higher probability of presenteeism whereas this probability falls with the number of years spent in education.

However, extant empirical studies, which are often conducted by occupational health researchers, are usually not based on a distinct theoretical model $\left.\right|^{2}$ Even from the side of economists, theoretical modelling of presenteeism is scarce, with no more than two extant studies, one of which even predicts that there is no presenteeism in equilibrium. Extending the Barmby et al. (1994) efficiency wage model of absenteeism, Brown and Sessions (2004) demonstrate that by varying the eligibility criterion for reduced firmfinanced sick pay employers can induce workers to either engage in absenteeism or in presenteeism. Based on a principle-agent model with perfect information but incomplete

$\overline{2}$ From the perspective of occupational medicine, Hansen and Andersen (2008, p. 957) lament that "it is not possible to embark from one comprehensive theory of sickness presenteeism from which one could derive a set of hypotheses to be tested." Johns (2010) offers some prescriptions for theory building on presenteeism, and Aronsson and Gustafsson (2005) and Gosselin et al. (2013) sketch a rough conceptual framework. 
contracting, Chatterji and Tilley (2002) show that firms are willing to pay more than the statutory sick pay to prevent ill workers with reduced productivity to come to work. Hence, in equilibrium employees do not engage in presenteeism. Neither of these models is consistent with the fact that typically within a firm some workers show presenteeism while at the same time others engage in absenteeism. What is more, the conclusions of both models are driven by the assumption that absent workers receive reduced sick pay (so that the firm can design optimal wage-sick pay contracts). This assumption neglects important institutional constraints, for instance that firms may not have discretion to alter eligibility criteria for sick pay or that the law may prescribe $100 \%$ sick pay for a certain amount of time, as is the case in many western welfare states.

Against this background, this paper contributes to the literature mainly in two ways: First, we develop a novel theoretical model of presenteeism (and absenteeism) that allows for full continuation of wage payments to sick workers and assumes that workers' dismissal probability rises with absence from work. In this model, paying higher wages provides incentives to show up at work and consequently employers use wages to incentivise workers. With two types of workers and imperfect information of employers on workers' disutility from workplace attendance, we show that firms set a wage such that sick workers get higher incentives than under perfect information and thus engage in presenteeism, whereas at the same time healthy workers receive lower incentives, resulting in absenteeism. This model enables us to derive several testable hypotheses on potential determinants of presenteeism such as workers' health status, working environment, tenure, and skill level (see Section 2). Our second, empirical contribution consists in testing these hypotheses using a large representative data set for Germany that includes specific questions on the prevalence of presenteeism (described in Section 3). We show that presenteeism plays an important role in Germany and find that the patterns in the data are largely consistent with the predictions of our model (see Section 4). We conclude that the phenomenon of presenteeism can well be analysed and explained economically, even if there exist a number of further, psychological, sociological, and medical determinants of presenteeism (Section 5). 


\section{An economic model of worker presenteeism}

In the following, we will set up a simple economic model of work attendance in which workers choose their workplace presence depending on their disutility from workplace attendance that is unobservable to their employer. Using wages to incentivise workers, employers provide too strong incentives for sick workers and too low incentives for healthy workers to show up at the workplace, resulting in an equilibrium with presenteeism and absenteeism coexisting.

\subsection{The starting point: the basic one-type model}

Consider a risk-neutral worker employed by a firm with $e \in[0,1]$ denoting the share of contractual working time the worker is present at her workplace. For every $e$ we assume the worker to suffer disutility $\operatorname{costs} \kappa e^{\psi}$ with $\kappa>0$ and $\psi>1$, so attendance at work reflects the effort exerted by the worker. Disutility costs are strictly increasing and disproportionately so in effort, and their level depends on the scale parameter $\kappa>0$ reflecting worker characteristics, like health status, that cause workplace attendance to be costly to the worker. Given that attendance at work is costly to the worker, she naturally tries to minimise it.

On the other hand, exerting effort benefits the worker because we assume that, depending on the worker's workplace absence, the firm is free to dismiss the worker with dismissal probability $1-e^{\delta} \in[0,1], \delta>0$, which strictly decreases with the worker's attendance at work. This latter assumption reflects that in order to successfully dismiss a worker the firm must be able to convince labour courts that the worker's absence from her workplace actually reflects either culpable behaviour (i.e. shirking) or chronic disease and hence the dismissal is lawful for one of these reasons. In the following, we will assume that $0<\delta<1$, i.e. that the dismissal probability is not only strictly decreasing but also strictly convex in workplace attendance. That is, we assume a dismissal to be disproportionately more likely if the worker's absence increases.

Now turn to the worker's remuneration. When staying with the firm for the entire period she receives some wage $w$, whereas she just gets some alternative income $b$ 
when being dismissed. Since the worker is risk-neutral, her optimal effort or workplace attendance maximises her expected income. Hence, her optimal effort level is

$$
e(w)=\underset{e}{\operatorname{argmax}}\left\{e^{\delta} w+\left(1-e^{\delta}\right) b-\kappa e^{\psi}\right\}= \begin{cases}\left(\frac{\delta(w-b)}{\psi \kappa}\right)^{\frac{1}{\psi-\delta}} & \text { if } w<b+\frac{\psi \kappa}{\delta} \\ 1 & \text { if } w \geqslant b+\frac{\psi \kappa}{\delta}\end{cases}
$$

Hence, we either have $w<b+\psi \kappa / \delta$ and arrive at an interior solution with $0<e(w)<1$, which follows from differentiating the globally concave maximand and setting its derivative to zero; or we have $w \geqslant b+\psi \kappa / \delta$, implying that the derivative of the maximand is positive for all $0<e<1$, and arrive at a corner solution with $e(w)=1$ I $^{3}$

Taking a closer look at the worker's effort supply function (1) under an interior solution with $0<e(w)<1$ we observe three things: (i) A higher wage $w$ translates into higher attendance at work as

$$
e^{\prime}(w)=\frac{\delta}{(\psi-\delta) \psi \kappa}\left(\frac{\delta(w-b)}{\psi \kappa}\right)^{\frac{\psi-\delta-1}{\psi-\delta}}>0
$$

for all $w>b$ (which will hold in equilibrium). The reason is simply that a higher wage means that a dismissal becomes more hurtful to the worker in terms of the associated income loss and that a higher wage thus provides her with an incentive to exert more effort. We will refer to this as the incentive effect of the wage, which is similar in spirit to the mechanism behind shirking variants of efficiency wage models (like Shapiro and Stiglitz, 1984) and relates to an earlier contribution by Barmby et al. (1994) analysing the interplay between worker absenteeism and firms' efficiency wages. (ii) A higher level of disutility from workplace attendance $\kappa$, e.g. due to acute illness of the worker or a permanent deterioration of her health status, renders effort more costly to the worker, ceteris paribus, and thus decreases attendance at work. (iii) If $\psi>\delta+\frac{1}{2}$, the incentive

3 Note that we can safely ignore the corner solution with complete workplace absence, i.e. $e=0$. To rule out this solution, it suffices to show that any solution with $0<e \leqslant 1$ satisfies $e^{\delta} w+\left(1-e^{\delta}\right) b-\kappa e^{\psi}>b$ or, equivalenty, $e<[(w-b) / \kappa]^{\frac{1}{\psi-\delta}}$. As is immediately clear from $\left.\sqrt{1}\right), e(w)<[(w-b) / \kappa]^{\frac{1}{\psi-\delta}}$ for all $\psi>\delta$, which holds by assumption. We will further see later, when deriving the firm's optimal wage-setting behaviour, that we even have to impose $\psi>\delta+1$ to obtain an equilibrium that is interesting from an economic point of view. Thus, the corner solution with $e=0$ is irrelevant indeed. 
effect of the wage is lower for workers with higher disutility from workplace attendance, i.e. $\partial e^{\prime}(w) / \partial \kappa<0$. That is, using wages to incentivise a worker is less effective for a worker with high disutility than for a worker with low disutility. As we will see, for the firm's profit maximisation problem to give a solution different from the trivial outcome with complete workplace presence for all possible parameter combinations $\psi>\delta+1$ even needs to hold. Hence, the incentive effect will always be lower for high-disutility compared to low-disutility workers.

Next, turn to the firm which has to decide on the worker's remuneration $w$. The worker's output is assumed to depend on her workplace presence $e$. For simplicity, we assume it to be proportional to $e$ with output being ae and the scale parameter $a>0$ reflecting worker characteristics, like the skill level, that affect the worker's overall productivity. In that we assume away, merely for tractability, that factors which affect the level of the worker's disutility costs, like the worker's health status, may also have a direct effect on her productivity ${ }^{4}$ Similarly, we abstract from adverse effects on co-workers' productivity levels that may either stem from complementarities in the production technology (e.g. Weiss, 1985; Coles and Treble, 1993) or infectious diseases (e.g. Skåtun, 2003; Barmby and Larguem, 2009). When deciding on the worker's wage the firm takes her effort supply behaviour into account. Thus, its profit-maximising wage is given by

$$
w^{*}=\underset{w}{\operatorname{argmax}}\{a e(w)-w\}= \begin{cases}b+\frac{\psi \kappa}{\delta}\left(\frac{a \delta}{(\psi-\delta) \psi \kappa}\right)^{\frac{\psi-\delta}{\psi-\delta-1}} & \text { if } a<\frac{(\psi-\delta) \psi \kappa}{\delta} \\ b+\frac{\psi \kappa}{\delta} & \text { if } a \geqslant \frac{(\psi-\delta) \psi \kappa}{\delta}\end{cases}
$$

For $a<(\psi-\delta) \psi \kappa / \delta$ the solution follows from differentiating the maximand and setting its derivative to zero, provided that $\psi>\delta+1$ which is necessary for the second-order condition

$4 \quad$ Yet, this simplification is harmless and does not affect our findings as long as the adverse effect on productivity is not too high and the firm thus still wants the worker to show up at work. In that case, the firm would still incentivise the sick worker, though to a somewhat lower extent (as the marginal revenue of a wage increase is now lower). If, on the other hand, the adverse effect on productivity is very large, the firm does not want the sick worker to show up at work at all, and incentivising her via wages gets pointless as worker and firm interests coincide. This case is investigated by Chatterji and Tilley (2002) who show that in this situation firms have an incentive to pay sick pay above the statutory minimum to prevent sick workers from showing up at work. 
of the firm's maximisation problem to hold $!^{5}$ On the other hand, for $a \geqslant(\psi-\delta) \psi \kappa / \delta$ the solution follows from the observation that paying $w^{*}=b+\psi \kappa / \delta$ already induces full workplace presence, see equation (1), so that paying more than that cannot be optimal. Plugging the firm's wage rule (3) into the worker's effort supply function (1) yields the worker's equilibrium workplace presence

$$
e^{*}= \begin{cases}\left(\frac{a \delta}{(\psi-\delta) \psi \kappa}\right)^{\frac{1}{\psi-\delta-1}} & \text { if } a<\frac{(\psi-\delta) \psi \kappa}{\delta}, \\ 1 & \text { if } a \geqslant \frac{(\psi-\delta) \psi \kappa}{\delta} .\end{cases}
$$

Inspecting the firm's wage rule (3) and the resulting optimal workplace attendance of the worker (4) we find three things: (i) The firm's optimal wage offer exceeds the alternative income $b$, for otherwise a dismissal would not be hurtful to the worker and she would have no incentive to exert any effort. This mirrors the incentive effect of the wage on attendance at work. (ii) Under an interior solution with $0<e^{*}<1$, the optimal wage and workplace attendance decrease in the disutility of effort $\kappa$. This reflects our previous finding that incentivising high-disutility individuals is less effective. (iii) Further, under an interior solution, the optimal wage and attendance at work increase in worker productivity $a$. This holds because workplace absence of a more productive worker has a more detrimental effect on firm profits, and thus the firm wants high-productivity individuals to show a higher workplace attendance, ceteris paribus.

\subsection{The reference world: the two-type model under perfect information}

We now use the modelling framework introduced in the previous section to study worker presenteeism and absenteeism. To do so in the simplest way, we first enrich our model by introducing two types of workers who differ in their disutility from workplace attendance.

5 If, otherwise, $\delta<\psi<\delta+1$ were to hold, firm profits would be strictly increasing in the wage for all $w<b+\psi \kappa / \delta$ and all parameter combinations, thereby yielding a corner solution with $w^{*}=b+\psi \kappa / \delta$ in any case. Intuitively, this would be the trivial situation in which the adverse effect of workplace attendance on the dismissal probability globally dominates the adverse effect on the worker's disutility costs. As a consequence, a higher wage would disproportionately raise the worker's effort, and the firm would set the wage such high that the worker shows full workplace attendance in any case. 
Without loss of generality, we assume that a share $\theta$ of workers have high disutility $\kappa>1$, e.g. due to acute or chronic illness, ("H-types") whereas the remaining workers have a low disutility at unity ("L-types"). For the moment, we further restrict attention to a situation with interior solutions, i.e. incomplete workplace attendance, for both types of workers. Following the derivation of the effort supply function (1) in the one-type model, workers thus differ in their effort supply behaviour with the supply function of type $i=H, L$ being

$$
e_{H}(w)=\left(\frac{\delta(w-b)}{\psi \kappa}\right)^{\frac{1}{\psi-\delta}} \text { and } \quad e_{L}(w)=\left(\frac{\delta(w-b)}{\psi}\right)^{\frac{1}{\psi-\delta}}
$$

Hence, other things being equal $H$-type workers show a larger absence from their workplace than $L$-type workers.

In the first best, i.e. under perfect information, the firm can observe the particular type of every worker and is thus able to implement a type-specific wage policy along the lines of the wage-setting rule in the one-type model (3) taking into account type-specific effort supply (5). The optimal wage for type $i=H, L$ therefore is

$$
w_{H}^{*}=b+\frac{\psi \kappa}{\delta}\left(\frac{a \delta}{(\psi-\delta) \psi \kappa}\right)^{\frac{\psi-\delta}{\psi-\delta-1}} \text { and } \quad w_{L}^{*}=b+\frac{\psi}{\delta}\left(\frac{a \delta}{(\psi-\delta) \psi}\right)^{\frac{\psi-\delta}{\psi-\delta-1}}
$$

Plugging the respective wage-setting rule (6) into the respective effort supply function (5) results in workplace attendance of

$$
e_{H}^{*}=\left(\frac{a \delta}{(\psi-\delta) \psi \kappa}\right)^{\frac{1}{\psi-\delta-1}} \text { and } \quad e_{L}^{*}=\left(\frac{a \delta}{(\psi-\delta) \psi}\right)^{\frac{1}{\psi-\delta-1}}
$$

As is unsurprising given the discussion of our basic one-type model in Section 2.1, in the first best $H$-types receive lower wages and are more often absent from work than $L$-types because when incentivsing workers the firm takes into account that wages are a less effective incentive device for workers with high disutility from workplace attendance ${ }^{6}$ We will take this first-best solution $\left(e_{H}^{*}, e_{L}^{*}\right)$ as our point of reference in the following

$6 \quad$ Again, in our model high-disutility workers are only less incentivised because wage increases are a less effective incentive device for high-disutility than for low-disutility workers. If high-disutility workers were also less productive on the job than low-disutility workers, this would further add to the firm's reluctance to use wages to incentivise its high-disutility workers. 
where we will relax the assumption of perfect information on the firm's side and will derive a second-best solution $\left(\tilde{e}_{H}, \tilde{e}_{L}\right)$ when workers are better informed on their disutility costs than the firm. In particular, we define worker presenteeism as a situation where attendance at work is larger than in the first best with optimal type-specific incentives in place, i.e. if $\tilde{e}_{i}>e_{i}^{*}$, and worker absenteeism as the opposite case, i.e. if $\tilde{e}_{i}<e_{i}^{*}$. Defining presenteeism and absenteeism in this way makes conceptionally clear in what dimension these are actually inefficient. When engaging in presenteeism workers exert inefficiently high effort, whereas under absenteeism effort is inefficiently low due to the fact that workers cannot credibly reveal their true costs of being present at the job to the firm: 7

\subsection{Worker presenteeism and absenteeism: the two-type model under imperfect information}

We now relax our assumption of perfect information to derive worker types' optimal attendance at work under the second best and suppose a situation where the firm just knows the share $\theta$ of $H$-types among workers but not whether a particular worker has a high or a low disutility from workplace attendance. Note that we restrict attention to the case where all of the firm's workers receive the same wage independent of their attendance at work. Hence, unlike Chatterji and Tilley (2002) and Brown and Sessions (2004), we do not allow for differing sick pay for absent workers. We argue that using reduced sickness pay to incentivise workers is implausible given the institutional setting in many western welfare states. In Germany, for instance, there is obligatory full continuation of payments in case of sickness for the first six weeks (and reduced sick pay out of the public health insurance afterwards). We further rule out attendance premia for workers with high attendance at work. Here our omission is merely empirical: Only few firms pay attendance premia in the German labour market, so that this restriction may seem plausible 8

$7 \quad$ With this definition of absenteeism in place, it is also clear that absenteeism does not coincide with mere workplace absence or mere shirking, but reflects that workers shirk to a greater extent than under optimal incentives.

8 Note also that we abstract from the possibility that firms may offer contracts with different wageattendance premium combinations to induce self-selection of worker types into contracts. The latter 
Under imperfect information, rather than knowing the particular type of worker the firm just knows that a particular worker has probability $\theta$ of facing high costs from workplace attendance, whereas with probability $1-\theta$ she has a low disutility. The wage chosen to maximise expected profits is therefore

$$
\begin{aligned}
\tilde{w} & =\underset{w}{\operatorname{argmax}}\left\{a\left[\theta e_{H}(w)+(1-\theta) e_{L}(w)\right]-w\right\} \\
& =b+\frac{\psi}{\delta}\left[\left(\theta \kappa^{-\frac{1}{\psi-\delta}}+1-\theta\right)\left(\frac{a \delta}{(\psi-\delta) \psi}\right)\right]^{\frac{\psi-\delta}{\psi-\delta-1}} .
\end{aligned}
$$

Comparing $\tilde{w}$ to the first-best wages $w_{H}^{*}$ and $w_{L}^{*}$ in (6) we see at once that $w_{H}^{*}<\tilde{w}<w_{L}^{*}$, and we are thus prepared to find that second-best incentives for workplace attendance are stronger for $H$-types and weaker for $L$-types than under perfect information. Formally, we see this from plugging the firm's second-best wage rule (8) into (5) to get

$$
\begin{aligned}
& \tilde{e}_{H}=\kappa^{-\frac{1}{\psi-\delta}}\left(\theta \kappa^{-\frac{1}{\psi-\delta}}+1-\theta\right)^{\frac{1}{\psi-\delta-1}}\left(\frac{\alpha \delta}{(\psi-\delta) \psi}\right)^{\frac{\psi-\delta}{\psi-\delta-1}}=\underbrace{\left(\theta+(1-\theta) \kappa^{\frac{1}{\psi-\delta}}\right)^{\frac{1}{\psi-\delta-1}}}_{>1} e_{H}^{*}, \\
& \tilde{e}_{L}=\left(\theta \kappa^{-\frac{1}{\psi-\delta}}+1-\theta\right)^{\frac{1}{\psi-\delta-1}}\left(\frac{\alpha \delta}{(\psi-\delta) \psi}\right)^{\frac{\psi-\delta}{\psi-\delta-1}}=\underbrace{\left(\theta \kappa^{-\frac{1}{\psi-\delta}}+1-\theta\right)^{\frac{1}{\psi-\delta-1}}}_{<1} e_{L}^{*},
\end{aligned}
$$

where we made use of the first-best effort levels $e_{H}^{*}$ and $e_{L}^{*}$ from (7). Hence, we arrive at presenteeism for $H$-type workers with $\tilde{e}_{H}>e_{H}^{*}$ and absenteeism for $L$-type workers with $\tilde{e}_{L}<e_{L}^{*}$. The reason for this finding is that workers cannot credibly reveal their disutility from workplace attendance to the firm, whereas the firm sets wages to incentivise the "average" worker in the population which results in too high incentives for $H$-types and too low incentives for $L$-types relative to the first best.

Note that we arrived at this outcome by restricting attention to interior solutions with both $L$-types and $H$-types choosing incomplete attendance at work in both the first-best and the second-best equilibrium. In terms of the model's parameters, this corresponds to

would perfectly make sense in an environment where workers know their disutility from workplace attendance before signing the contract. This may be plausible for permanent factors affecting worker disutility, like disability (though these should arguably be, at least to some extent, observable to the firm). Yet, if we think of changes in the disutility after signing the contract, such as those due to acute or chronic deterioration of the worker's health status during an ongoing job (and this is what we are mainly interested in), this kind of worker self-selection gets inviable. 
the case $a<(\psi-\delta) \psi / \delta$. Yet, if $a \geqslant(\psi-\delta) \psi / \delta$, there are two further solutions: (i) For $(\psi-\delta) \psi / \delta \leqslant a<\bar{a}$ with some threshold productivity $\bar{a} L$-types choose full workplace attendance in the first best and incomplete attendance in the second best, where they receive a lower wage, i.e. $\tilde{e}_{L}<e_{L}^{*}=1$, whilst $H$-types have $e_{H}^{*}<\tilde{e}_{H}<1$ as before. So in this solution, $H$-types still show presenteeism, and $L$-types engage in absenteeism. (ii) If instead $a \geqslant \bar{a}$, the firm sets first-best and second-best wages such high that $L$ types choose full workplace attendance both in the first and the second best and thus show neither absenteeism nor presenteeism.9 In this case, the model does not generate absenteeism and presenteeism as coexisting equilibrium outcomes. This obviously is an uninteresting result when trying to explain the observed coexistence of worker absenteeism and presenteeism, so we impose $a<\bar{a}:^{10}$

\subsection{Presenteeism, absenteeism, and worker heterogeneity}

Next, consider how the predictions of our two-type model change if we alter one of the model's core parameters related to worker heterogeneity. We will analyse three different cases: (i) an increase in $\delta$ translating into a larger dismissal probability $1-e^{\delta}$ for every level of workplace attendance $0<e<1$, (ii) an increase in worker productivity $a$, and (iii) an increase in the share of high-disutility workers $\theta$. To keep the exposition as simple as possible, we will discuss these changes in the case of incomplete workplace attendance of both $L$-types and $H$-types in the first-best and second-best equilibria, i.e. for $a<(\psi-\delta) \psi / \delta$, though results would not change qualitatively as long as $a<\bar{a}$.

First, consider what happens if the parameter $\delta$ increases, i.e. a worker with incomplete attendance at work is more likely to be dismissed. Note that if $\partial\left(\tilde{e}_{H} / e_{H}^{*}\right) / \partial \delta>0$ this implies $\partial \tilde{e}_{H} / \partial \delta>\partial e_{H}^{*} / \partial \delta$ and thus an increase in $H$-types' presenteeism. On the

$9 \quad$ As in the second best $L$-types choose full workplace presence if and only if $\tilde{w} \geqslant b+\psi / \delta$, the threshold productivity directly follows from 88 as $\bar{a}=(\psi-\delta) \psi /\left[\left(\theta \kappa^{-\frac{1}{\psi-\delta}}+1-\theta\right) \delta\right]$.

10 Note that in the two-type model neither $L$-types nor $H$-types choose full workplace attendance in the second best. This seems at odds with the empirical observation that a non-trivial share of individuals report no workplace absence at all. In order to reconcile this empirical fact, one could readily extend the model by introducing a third type of workers. In this extended model, a possible equilibrium would have workers with low disutility choosing full workplace attendance, workers with medium disutility engaging in absenteeism, and workers with high disutility showing presenteeism. As such an extended model would not change our insights, we shall stick to the much more tractable two-type model in the following. 
other hand, if $\partial\left(\tilde{e}_{L} / e_{L}^{*}\right) / \partial \delta<0$ this implies $\partial \tilde{e}_{L} / \partial \delta<\partial e_{L}^{*} / \partial \delta$, and therefore $L$-types' absenteeism rises ${ }^{11}$ Using both worker types' optimal second-best workplace attendance (9) and defining $\tau \equiv \theta+(1-\theta) \kappa^{\frac{1}{\psi-\delta}}$ with $\tau>1$ and $\chi \equiv \theta \kappa^{-\frac{1}{\psi-\delta}}+1-\theta$ with $0<\chi<1$ as shorthands, we find

$$
\begin{aligned}
& \frac{\partial \frac{\tilde{e}_{H}}{e_{H}^{*}}}{\partial \delta}=\tau^{\frac{1}{\psi-\delta-1}}\left(\frac{\ln \tau}{(\psi-\delta-1)^{2}}+\frac{(1-\theta) \kappa^{\frac{1}{\psi-\delta}} \ln \kappa}{(\psi-\delta-1)(\psi-\delta)^{2} \tau}\right)>0 \quad \text { and } \\
& \frac{\partial \frac{\tilde{e}_{L}}{e_{L}^{*}}}{\partial \delta}=\chi^{\frac{1}{\psi-\delta-1}}\left(\frac{\ln \chi}{(\psi-\delta-1)^{2}}-\frac{\theta \kappa^{-\frac{1}{\psi-\delta}} \ln \kappa}{(\psi-\delta-1)(\psi-\delta)^{2} \chi}\right)<0,
\end{aligned}
$$

so that both presenteeism of $H$-types and absenteeism of $L$-types get more pronounced.

Second, turn to worker productivity $a$, which may for instance vary across workers with different skill levels. As we discussed earlier, the higher is workers' productivity the higher is the firm's wage in the first best as incentivising high-productivity workers pays off more. On the other hand, in the second best the wage will increase by less than in the first best because of the $H$-type workers in the workforce for whom the wage is a less effective incentive device. Hence, the overall effect of a change in worker productivity on worker absenteeism and presenteeism is not obvious. Using (9) we find

$$
\begin{aligned}
\frac{\partial \tilde{e}_{H}}{\partial a} & =\underbrace{\left(\theta+(1-\theta) \kappa^{\frac{1}{\psi-\delta}}\right)^{\frac{1}{\psi-\delta-1}}}_{>1} \frac{\partial e_{H}^{*}}{\partial a} \quad \text { and } \\
\frac{\partial \tilde{e}_{L}}{\partial a} & =\underbrace{\left(\theta \kappa^{-\frac{1}{\psi-\delta}}+1-\theta\right)^{\frac{1}{\psi-\delta-1}}}_{<1} \frac{\partial e_{L}^{*}}{\partial a},
\end{aligned}
$$

so that an increase in worker productivity causes both presenteeism of $H$-types and absenteeism of $L$-types to rise.

Finally, consider a change in the distribution of worker types in the firm's workforce, i.e. an increase in the share $\theta$ of $H$-type workers. Having a look at the firm's second-best wage rule (8) we see at once that this depresses the wage paid by the firm because there are more $H$-types for whom the wage is a less effective device for providing incentives. On the other hand, first-best wages (6) and workplace attendance levels (7) remain unaltered.

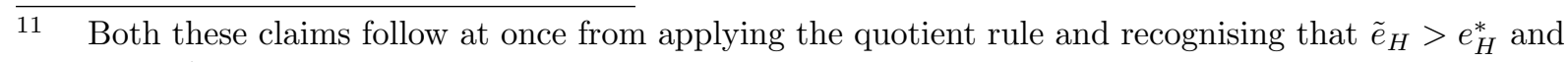
$\tilde{e}_{L}<e_{L}^{*}$. 
We thus expect presenteeism of $H$-types to fall and absenteeism of $L$-types to rise. Using (9) we indeed arrive at these predictions, i.e.

$$
\begin{aligned}
& \frac{\tilde{e}_{H}}{\partial \theta}=\frac{\left(\theta+(1-\theta) \kappa^{\frac{1}{\psi-\delta}}\right)^{\frac{2+\delta-\psi}{\psi-\delta-1}}\left(1-\kappa^{\frac{1}{\psi-\delta}}\right)}{\psi-\delta-1} e_{H}^{*}<0 \quad \text { and } \\
& \frac{\tilde{e}_{L}}{\partial \theta}=\frac{\left(\theta \kappa^{-\frac{1}{\psi-\delta}}+1-\theta\right)^{\frac{2+\delta-\psi}{\psi-\delta-1}}\left(\kappa^{-\frac{1}{\psi-\delta}}-1\right)}{\psi-\delta-1} e_{L}^{*}>0 .
\end{aligned}
$$

As our previous theoretical predictions all rest on a given distribution of worker types, equation 12 implies that it will be crucial in our empirical analysis to condition on factors likely to reflect a different composition of workers in terms of their disutility from workplace attendance, that is controlling for professional field, sex, age, and family status.

\subsection{Hypotheses}

Our theoretical model thus provides us with the following set of hypotheses on sickness presenteeism which we are going to test in our empirical analysis: 12

(H1) We expect workers to differ in presenteeism, which reflects differences in the disutility from workplace presence among workers, compare equation (9). In particular, workers with bad health or stressful working environments should show (more pronounced) presenteeism.

(H2) Workers with higher tenure, who are typically subject to more pronounced dismissal protection, should show less presenteeism, see equation 10 .

(H3) Worker presenteeism should be more relevant for high-skilled workers whose productivity is arguably larger, compare equation (11).

\section{$3 \quad$ Data and descriptive evidence}

To confront our theoretical predictions with data, we use the BIBB/BAuA Employment Survey of the Working Population on Qualification and Working Conditions in Germany

12 Although our model also provides clear predictions concerning absenteeism, we will not pursue these here, merely because (in contrast to presenteeism) our survey data do not contain a suitable question on absenteeism as defined in our model, but only on mere workplace absence (see also fn. 7). 
2012 (Hall et al., 2014; see Rohrbach-Schmidt and Hall, 2013, for a detailed description). This survey contains information on a representative cross-section of 20,036 individuals from the German active labour force population, i.e. individuals who are at least 15 years old and work at least 10 hours per week for pay. The data set is especially suitable for our purposes because it provides detailed information both on the incidence and extent of presenteeism as well as on the health status of individuals and the strains they experience at the workplace. Additionally, there is information on individuals' socio-demographic characteristics and on the professional fields they are active in. That said, a limitation of the data set is that it is cross-sectional, so that we are not able to take unobserved heterogeneity into account. Still, we are not aware of panel data containing similarly adequate or better information regarding our research questions.

We restrict our estimation sample to workers employed in the private sector as the risk of job loss, which creates incentives for presenteeism in our model, is much less a matter for workers in the public sector (including civil servants, who are virtually impossible to dismiss). We also exclude individuals older than 65 years and those who report working more than 60 hours per week (which is the legally binding maximum of working hours in Germany). Finally, we drop some observations with suspect data on presenteeism, namely more than 220 days of presenteeism per year (about $0.1 \%$ of all observations), 99 presenteeism days per year (missings are coded as 999 in the data set), or more than 365 days of sickness (at home plus at work) per year. In the end, we obtain a sample of 11,016 observations with complete data on all relevant covariates.

The measure of presenteeism we use is based on two survey questions on the incidence and extent of attending work despite bad health. First, respondents were asked: "In the last 12 months, did you ever go to work although you should better have called in sick due to your state of health?" The framing of this question seems quite fortunate as it corresponds well with our theoretical understanding of presenteeism, where individuals maximize utility by showing presenteeism in a second-best world, but nevertheless sense that in a first-best world they "should better have called in sick." When answering in the affirmative, respondents were subsequently asked: "How many workdays were that all in all?" 
Table 1 shows that in our sample the average number of workdays in the last 12 months when individuals went to work despite being sick, henceforth referred to as presenteeism days, amounts to 6.3 days. The standard deviation of 14.2 days indicates that there is a lot of cross-sectional variation. About one-half (55\%) of individuals show one or more days of presenteeism, so by implication $45 \%$ of employees never attend work when feeling sick $\sqrt{13}$ Presenteeism days also vary widely within the group of workers with strictly positive days of presenteeism. Those workers who show at least some presenteeism have 11.6 presenteeism days on average with a standard deviation of 17.6 days. For this group, the median value of presenteeism amounts to 6 days, while $90 \%$ of these workers report no more than 20 presenteeism days per year. In sum, the distribution of presenteeism days appears to be heavily skewed right with a mass point at zero. Still, presenteeism takes on a wide range of possible values ranging from 0 to 220 days. We account for these distributional features in our econometric analysis by estimating different models for the conditional mean and, additionally, separately investigating the mass point at zero.

Turning to the crucial explanatory variables of our study, we capture workers' disutility from workplace attendance (unobservable to the employer) by their subjective health status and by the number of working conditions they regard as stressful. Health status is reported on a five-point Likert scale, ranging from excellent to bad. Most individuals $(55 \%)$ assess their health status as "good" and about a third of individuals (31\%) even report "very good" or "excellent" health (see Table 1). This leaves a share of $14 \%$ of workers with a health status that is being perceived as "not so good" or "bad".

As a second measure of their disutility at work, we use the number of a total of 23 unpleasant working conditions individuals feel stressed by. Examples for such unpleasant working conditions are working exposed to cold, heat, moisture, humidity, or draughts, handling of hazardous substances, working under a lot of pressure, or performing repetitive

13 Clearly, we cannot rule out recall bias concerning the incidence and extent of presenteeism. That said, our figures lie within the range reported for Germany in previous studies based on smaller samples covering the entire economy and not just the private sector. According to the European Working Conditions Survey 2010 (Eurofound, 2012), 39\% of employees engage in presenteeism, whereas this share is over $70 \%$ in a health survey from 2008 analysed by Vogt et al. (2009) and even reaches $88 \%$ in a small and not representative pilot study by Preisendörfer (2010) where the respective question is not restricted to presenteeism in the last 12 months. 
Table 1: Descriptive statistics

\begin{tabular}{|c|c|c|}
\hline & Mean & St.dev. \\
\hline \multicolumn{3}{|l|}{ Presenteeism during the past 12 months } \\
\hline Number of workdays & 6.30 & 14.23 \\
\hline At least once (dummy) & 0.55 & 0.50 \\
\hline Number of workdays conditional on positive & 11.55 & 17.61 \\
\hline \multicolumn{3}{|l|}{ Health status } \\
\hline Excellent (dummy) & 0.09 & 0.28 \\
\hline Very good (dummy) & 0.22 & 0.42 \\
\hline Good (dummy) & 0.55 & 0.50 \\
\hline Not so good (dummy) & 0.12 & 0.32 \\
\hline Bad (dummy) & 0.02 & 0.14 \\
\hline Number of stressful working conditions & 2.03 & 2.72 \\
\hline Tenure at current employer (years) & 12.10 & 10.60 \\
\hline \multicolumn{3}{|l|}{ Qualification } \\
\hline High-skilled (dummy) & 0.28 & 0.45 \\
\hline Medium-skilled (dummy) & 0.65 & 0.48 \\
\hline Low-skilled (dummy) & 0.07 & 0.26 \\
\hline Working hours per week & 37.55 & 10.72 \\
\hline Female (dummy) & 0.50 & 0.50 \\
\hline Age (years) & 44.69 & 10.55 \\
\hline Partner in household (dummy) & 0.63 & 0.48 \\
\hline Child(ren) in household (dummy) & 0.32 & 0.47 \\
\hline Observations & \multicolumn{2}{|c|}{11,016} \\
\hline
\end{tabular}

Notes: The data set used is the BIBB/BAuA Employment Survey 2012.

work. Clearly, working conditions as such are at least partly observable by the employer, while our theory suggests that it is unobserved differences in disutility at work that cause presenteeism. Fortunately, the survey not only asked whether certain working conditions were present, but also whether respondents actually felt stressed by these working conditions. While an employer may observe working conditions, she is probably not able to observe how different workers experience the same working conditions. Therefore, in line with our theoretical model, we count the number of working conditions by which individuals feel stressed ${ }^{14}$ On average, this number amounts to 2.0 in our sample (with a standard deviation of 2.7 ; see Table 1 ).

14 That said, our results are robust to using the total number of unpleasant working conditions present at the workplace no matter whether individuals regard them as stressful. 
As hypothesised above (see Section 2), we also expect tenure and the skill level of workers to be related to presenteeism. The tenure at the current employer amounts to 12.1 years on average in our sample (see Table 1). For the skill level, we use three dummies (high-skilled, medium-skilled, low-skilled) based on the highest professional qualification. Most workers (65\%) are medium-skilled, i.e. they have completed vocational training. $28 \%$ of respondents have an academic degree or a qualification as master craftsman, state certified technician, business administrator, or the like (high-skilled group). Our reference group are low-skilled workers without any professional qualification (7\% in the sample; see Table 1).

To get a first impression of the correlates of presenteeism, Table 2 displays the incidence and extent of presenteeism separately for several subgroups of workers. First of all, rows 2 and 3 of Table 2 compare workers in better health (i.e. health status is being perceived as excellent, very good, or good) with those in worse health (not so good, bad). About one-half of those in better health have been present at work although they should have better called in sick at least once, and the average number of presenteeism days amounts to 4.6 for this group. This is in stark contrast to the presenteeism of workers with worse health. About $80 \%$ of those with worse health have shown presenteeism, and the average number of presenteeism days is almost four times higher in this group than in the group with better health (namely 16.7 days).

Table 2 also shows that $73 \%$ of those workers with above-average numbers of stressful working conditions engage in presenteeism, whereas this is only the case for $46 \%$ of those with stressful working conditions below average. Presenteeism days differ in similar fashion between both groups (see rows 4 and 5 of Table 2). In other words, someone who dislikes going to work much nevertheless goes to work more often when sick than someone who is more comfortable with her work. This finding appears counterintuitive at first, but is exactly what is predicted by our model and stated in hypothesis 1 . Thus, the relationships between both our measures of (health-related) disutility and presenteeism are well in line with our theoretical expectations ${ }^{15}$ They are in line with extant evidence by Oldenburg

$15 \quad$ An additional explanation for the positive correlation between health status and presenteeism could be that going to work when sick may exacerbate existing health problems. 
Table 2: Descriptive evidence on the correlates of presenteeism

\begin{tabular}{lcc}
\hline & $\begin{array}{c}\text { Presenteeism } \\
\text { incidence } \\
\text { (share in \%) }\end{array}$ & $\begin{array}{c}\text { Presenteeism } \\
\text { days } \\
\text { (mean) }\end{array}$ \\
\hline Overall & 54.6 & 6.3 \\
Better health (excellent, very good, good) & 50.4 & 4.6 \\
Worse health (not so good, bad) & 80.4 & 16.7 \\
Stressful working conditions above average & 73.4 & 10.6 \\
Stressful working conditions below average & 46.4 & 4.4 \\
Tenure with current employer above average & 52.3 & 6.2 \\
Tenure with current employer below average & 56.1 & 6.4 \\
High-skilled & 49.4 & 5.0 \\
Medium-skilled & 56.9 & 6.9 \\
Low-skilled & 54.0 & 6.1 \\
\hline Observations & \multicolumn{2}{c}{11,016} \\
\hline
\end{tabular}

Notes: The data set used is the BIBB/BAuA Employment Survey 2012.

(2012) who, in a descriptive analysis based on the same data set but somewhat different variables, also finds that presenteeism is positively related to bad health and uncomfortable working conditions.

Our second hypothesis states that workers with higher tenure will show lower presenteeism since they are better protected against dismissal than workers with low tenure. Comparing the group of workers with tenure above average with workers with below-average tenure, we find the expected bivariate correlation, but the difference in presenteeism between the two groups appears not to be very pronounced ( $52 \%$ at least once and 6.2 days vs. $56 \%$ at least once and 6.4 days on average; see rows 6 and 7 of Table 2).

Finally, comparing presenteeism between groups of different skill levels does not reveal a relationship with clear direction. The incidence and extent of presenteeism is somewhat higher in the medium-skilled group than in the low-skilled group, but is lowest in the high-skilled group (see rows 8 to 10 of Table 2). We see thus no supportive evidence for our third hypothesis in the univariate case.

Although these descriptive results are interesting, they do not take into account potential interdependencies, for instance that health status may be correlated with 
working conditions which are themselves correlated with skill level and so on. In particular, the above results are not conditional on working in a certain professional field within which it is more plausible that employers face a given distribution of worker types (i.e. a given $\theta$ in our theoretical model). To address these points, we now turn to a multivariate econometric analysis.

\section{Econometric evidence}

We are mainly interested in the average partial effects of our explanatory variables on the conditional expectation of presenteeism days, i.e. in the partial correlation between presenteeism days and health status, the number of stresses and strains at the workplace, tenure, and skill level. Additionally, we investigate the mass point of presenteeism days at zero. We thus present estimates of four different models in Table 3 .

As a starting point, we fit an OLS regression model of presenteeism days (column 1 of Table 3). This approach is feasible since we need not get the functional form perfectly right to obtain valid estimates of average partial effects. Yet, presenteeism days can only take on non-negative integer values, so it seems natural to also make use of a count data model. Therefore, we second present estimates of a Poisson model (column 2 of Table 3). The Poisson model for count data is sometimes discarded because the Poisson distribution imposes equality of variance and mean, a feature that data of presenteeism days typically do not show (see the discussion in Pedersen and Skagen, 2014). However, this objection overlooks that the Poisson quasi maximum likelihood estimator is robust to arbitrary misspecification of the variance (see, e.g., Wooldridge, 2010, ch. 18.2.2), a robustness property not shared by more sophisticated count data models like the Negative Binomial. ${ }^{16}$ Still, a limitation of the Poisson model is that it does not explicitly take the clustering at zero into account. As a third specification, we thus estimate a Tobit model (column 3 of Table 3), a convenient choice for modelling corner solution outcomes where the dependent variable takes on a wide range of possible values (see Wooldridge, 2010,

16 That said, our insights do not change when using a Negative Binomial model (results are available on request). 
ch. 17) ${ }^{17}$ In a fourth specification, we have a closer look at the mass point at zero. For this purpose, we estimate a Probit model for the incidence of presenteeism, i.e. showing presenteeism at least once (column 4 of Table 3$)^{18}$

In all four models, the explanatory variables are as follows. To test our first hypothesis, we include four health status dummies ("good" being the reference group) and the number of working conditions individuals feel stressed by. Tenure (in years) and two skill level dummies (reference group "low-skilled") serve to test hypotheses 2 and 3, respectively. As our model assumes a given distribution of worker types, and in order to control for heterogeneity across occupations, we condition on 54 professional fields (based on the classification by Tiemann et al., 2008), within which it is more plausible that employers face a given distribution of worker types, i.e. a given $\theta$. Additionally, we include working hours per week because it is probably less onerous to go to work in case of sickness when you do not have to be present that long and because workers with fewer working hours may have more working time flexibility and fewer working days per year. Finally, we add some standard socio-economic control variables, namely a sex dummy, age (in years), dummies for having a partner or child(ren) in the household, and 16 federal state dummies.

Table 3 shows that presenteeism is typically higher the worse health status, ceteris paribus. Being in very good or excellent health instead of good health is associated with about 2 to 4 presenteeism days less on average (depending on specification), while bad health goes along with 13 to 17 more presenteeism days on average, and these differences are statistically significant at the $1 \%$ level. These figures also highlight the economic significance of health status for the extent of presenteeism, given a mean of presenteeism days of about 6 (see Table1). Looking at the partial correlation of health and the incidence

17 An alternative model that explicitly takes the mass point at zero into account would be the ZeroInflated Negative Binomial (ZINB) model. However, the ZINB model is not really appropriate because it assumes that there exist "excessive zeros", i.e. that it is impossible to have strictly positive presenteeism days for some individuals. This would, for instance, be the case if some individuals were not in the workforce and so would necessarily have zero presenteeism days, whether sick or not. Since our sample comprises only individuals from the active labour force population, such excessive zeros are unlikely to occur. Anyhow, our insights do not change when estimating a ZINB model with health status additionally appearing in the inflation equation (results available on request).

18 However, we refrain from separately presenting a "second stage", i.e. average partial effects that are conditional on showing presenteeism at least once. Restricting the sample to those with strictly positive presenteeism days would be a case of endogenous sample selection, resulting in uninformative estimates (see, e.g., Elwert and Winship, 2014, Angrist and Pischke, 2009, pp. 99-102). 
Table 3: Econometric analysis of presenteeism

\begin{tabular}{|c|c|c|c|c|}
\hline \multirow[t]{2}{*}{ Dependent variable } & \multicolumn{3}{|c|}{ Presenteeism days } & \multirow{2}{*}{$\begin{array}{c}\text { incidence } \\
\text { Probit }\end{array}$} \\
\hline & OLS & Poisson & Tobit & \\
\hline \multicolumn{5}{|l|}{ Health status (reference: good) } \\
\hline \multirow[t]{2}{*}{ Excellent (dummy) } & $-2.446^{* *}$ & $-3.186^{* *}$ & $-3.804^{* *}$ & $-0.245^{* *}$ \\
\hline & $(0.264)$ & $(0.272)$ & $(0.262)$ & $(0.016)$ \\
\hline \multirow[t]{2}{*}{ Very good (dummy) } & $-2.141^{* *}$ & $-2.706^{* *}$ & $-2.779^{* *}$ & $-0.164^{* *}$ \\
\hline & $(0.201)$ & $(0.204)$ & $(0.212)$ & $(0.012)$ \\
\hline \multirow[t]{2}{*}{ Not so good (dummy) } & $8.059^{* *}$ & $7.141^{* *}$ & $6.875^{* *}$ & $0.188^{* *}$ \\
\hline & $(0.695)$ & $(0.646)$ & $(0.422)$ & $(0.013)$ \\
\hline \multirow[t]{2}{*}{ Bad (dummy) } & $17.328^{* *}$ & $13.236^{* *}$ & $13.489^{* *}$ & $0.164^{* *}$ \\
\hline & $(2.442)$ & $(1.872)$ & $(1.124)$ & $(0.032)$ \\
\hline \multirow[t]{2}{*}{ Number of stressful working conditions } & $0.827^{* *}$ & $0.504^{* *}$ & $0.723^{* *}$ & $0.041^{* *}$ \\
\hline & $(0.083)$ & $(0.045)$ & $(0.037)$ & $(0.002)$ \\
\hline \multirow[t]{2}{*}{ Tenure at current employer (years) } & $-0.046^{* *}$ & $-0.046^{* *}$ & $-0.032^{* *}$ & -0.001 \\
\hline & $(0.015)$ & $(0.014)$ & $(0.011)$ & $(0.001)$ \\
\hline \multicolumn{5}{|l|}{ Qualification (reference: low-skilled) } \\
\hline \multirow[t]{2}{*}{ High-skilled (dummy) } & 0.763 & 0.692 & 0.501 & -0.005 \\
\hline & $(0.537)$ & $(0.611)$ & $(0.443)$ & $(0.020)$ \\
\hline \multirow[t]{2}{*}{ Medium-skilled (dummy) } & $1.222^{*}$ & $1.067^{*}$ & $1.010^{* *}$ & $0.035^{*}$ \\
\hline & $(0.475)$ & $(0.449)$ & $(0.371)$ & $(0.018)$ \\
\hline \multirow[t]{2}{*}{ Working hours per week } & $0.101^{* *}$ & $0.103^{* *}$ & $0.101^{* *}$ & $0.004^{* *}$ \\
\hline & $(0.013)$ & $(0.014)$ & $(0.011)$ & $(0.001)$ \\
\hline \multirow[t]{2}{*}{ Female (dummy) } & $1.688^{* *}$ & $1.718^{* *}$ & $1.770^{* *}$ & $0.089^{* *}$ \\
\hline & $(0.327)$ & $(0.327)$ & $(0.252)$ & $(0.012)$ \\
\hline \multirow[t]{2}{*}{ Age (years) } & -0.018 & -0.018 & $-0.072^{* *}$ & $-0.006^{* *}$ \\
\hline & $(0.015)$ & $(0.015)$ & $(0.011)$ & $(0.001)$ \\
\hline \multirow[t]{2}{*}{ Partner in household (dummy) } & 0.110 & 0.079 & 0.131 & 0.011 \\
\hline & $(0.316)$ & $(0.294)$ & $(0.208)$ & $(0.010)$ \\
\hline \multirow[t]{2}{*}{ Child(ren) in household (dummy) } & 0.024 & -0.005 & 0.088 & 0.007 \\
\hline & $(0.306)$ & $(0.304)$ & $(0.225)$ & $(0.010)$ \\
\hline Professional field (54 dummies) & $p<0.01$ & $p<0.01$ & $p<0.01$ & $p<0.01$ \\
\hline Place of residence (16 state dummies) & $p=0.49$ & $p=0.43$ & $p=0.63$ & $p=0.22$ \\
\hline$R^{2}$ & 0.144 & 0.155 & 0.144 & 0.095 \\
\hline Number of observations & \multicolumn{4}{|c|}{11,016} \\
\hline
\end{tabular}

Notes: The data set used is the BIBB/BAuA Employment Survey 2012. Reported numbers are average partial effects with standard errors in parentheses (which are robust for the OLS and Poisson models). **/* indicates statistical significance at the $1 / 5 \%$ level. The reported $R^{2}$ is the squared correlation between actual and predicted outcomes. 
of presenteeism reveals a similar pattern (see column 4 of Table 3). Compared to good health status, the probability of having shown presenteeism at least once is lower by about 25 and 16 percentage points when having an excellent and very good health status, respectively, and is higher by 16 percentage points in case of bad health, ceteris paribus. Yet, we cannot claim causality here. The positive relationship identified may be due to a genuine causal effect but may also reflect that going to work when ill exacerbates existing health problems ${ }^{19}$

The average partial effects of the number of working conditions individuals regard as stressful also point in the expected direction and are statistically significant. Feeling stressed by one more unpleasant working condition goes along with about 0.5 to 0.8 additional presenteeism days on average and a 4 percentage points higher probability of having shown presenteeism. Taken together, these results provide strong support for hypothesis 1 that a higher (health-related) disutility at work is associated with more presenteeism.

Turning to our second hypothesis, the results in Table 3 show that ten more years at the current employer (which is approximately one standard deviation) are associated with at maximum 0.5 days less presenteeism per year on average, ceteris paribus. While this statistically significant finding is in line with our theoretical expectations, the small magnitude of the estimated average partial effects suggests that the practical relevance of tenure for presenteeism is not overly high. Moreover, looking at the incidence of presenteeism reveals that tenure is practically partially uncorrelated with the probability of showing presenteeism at least once. Again, simultaneity may play a role here. If workers who show more presenteeism obtain more stable jobs, higher presenteeism goes along with higher tenure, and our estimates for tenure would be biased towards finding no effect. Thus, our results probably provide a lower bound for the negative effect of better dismissal protection on presenteeism.

19 One could also argue that sick people show more presenteeism simply because being sick is necessary for showing presenteeism. And if the probability of attending work when sick were exogenously given, individuals with worse health would then show more presenteeism. This line of reasoning boils down to disregard presenteeism as a choice variable. In contrast, we argue that the patterns in the data can in fact be rationalised as the result of optimising behaviour. 
Our third hypothesis states that presenteeism should be higher for more skilled workers whose productivity can be expected to be higher. Yet, the estimates in Table 3 show that the relationship between workers' skill level and presenteeism is not that clear. In line with our hypothesis, medium-skilled workers display about 1 more presenteeism day than lowskilled workers and have a 3.5 percentage points larger probability of showing presenteeism at least once. On the other hand, high-skilled workers do not show more presenteeism than medium-skilled workers. What is more, the presenteeism of high-skilled workers is not even statistically significantly different from that of the low-skilled group, regardless of whether one looks at presenteeism days or incidence. A potential explanation for this finding consistent with our model is that while high-skilled workers are more productive and should thus show more presenteeism (see hypothesis 3), they are not as easy to replace as less skilled workers and so face a lower dismissal probability, resulting in lower presenteeism (see hypothesis 2). The average partial effects of the skill dummies could thus reflect a combination of these two opposing effects.

Concerning our control variables, the 54 professional field dummies are jointly statistically significant at the $1 \%$ level, and working hours are positively related to presenteeism, as expected. Presenteeism is more prevalent among women than men, ceteris paribus, and older workers are less likely to exhibit at least one day of presenteeism (whereas the negative relationship between presenteeism days and age is not robust across all specifications). Eventually, neither family status, firm size, nor place of residence are associated with either presenteeism incidence or extent.

All in all, the correlations identified in the data are indicative that presenteeism indeed arises due to differences in (health-related) disutility from workplace attendance that are unobservable to the firm, which is the theoretical explanation for presenteeism we propose in this paper. Also in line with our theoretical model, we find that better dismissal protection, captured by higher tenure, is associated with fewer days of presenteeism, although the economic significance of this relationship is not that marked. A further prediction of our model concerning the role of productivity and skills is not supported by the data, possibly because of confounding factors that bias our estimates for the average partial effects of tenure and skill level towards zero. 
Note that our insights still hold when performing a number of robustness tests. First, to rule out that our results are affected by a different behaviour of employees during their probation period, we excluded individuals with tenure of less than one year. Likewise, we excluded temporary workers who might behave differently than regularly employed workers. Second, we ran separate analyses for men and women. Third, we included ten firm size dummies as additional controls, which turned out to be statistically insignificant (we omitted firm size previously for a lack of theoretical justification and because including this information would reduce the sample size by more than 600 observations). Finally, using the number of unpleasant working conditions rather than the number of these working conditions regarded as stressful by workers also did not change our findings.

\section{Concluding remarks}

This paper has provided a theoretical and empirical investigation into the phenomenon of sickness presenteeism from an economic perspective. Using a large representative data set for Germany in 2012, we have shown that presenteeism is of substantial size: $55 \%$ of workers reported that they came to work at least once in the last 12 months when they should better have called in sick, with the average number of presenteeism days amounting to 6.3. We also have demonstrated that presenteeism can be interpreted as the result of optimising behaviour (under imperfect information) on the sides of employees and employers, and that various economic factors are related to the incidence and extent of presenteeism.

To this end, we first have developed a novel theory of presenteeism that takes account of institutional factors such as $100 \%$ sick pay and that is consistent with the fact that usually presenteeism and absenteeism coexist in the same firm. In our model, presenteeism arises when employers have imperfect information about the health status of their workers, and thus about workers' disutility from workplace attendance. As a consequence, firms set a wage such that sick workers get higher incentives than under perfect information and thus engage in presenteeism, whereas at the same time healthy workers get lower incentives, which results in absenteeism. This model has enabled us to derive several hypotheses 
on potential determinants of presenteeism. Testing these hypotheses econometrically, we have found that presenteeism is indeed related to unobservable differences in disutility from workplace attendance, proxied by employees' health status and by the number of unpleasant working conditions they feel stressed by. Also in line with our theoretical predictions, we have shown that better dismissal protection, captured by higher tenure, is associated with (slightly) fewer days of presenteeism.

Our theoretical and empirical insights demonstrate that the phenomenon of presenteeism can well be analysed and explained economically, even if the vast bulk of the extant literature consists of non-economic and mainly empirical approaches by researchers of occupational medicine. Although we have focused on a small number of variables derived from economic theory, we acknowledge that there exist a number of further, psychological, sociological, medical, or organisational determinants of presenteeism such as work attitudes and personality traits, working environments and relationships with colleagues, norms and presenteeism cultures, social dynamics, etc. (see the survey by Johns, 2010). In order to obtain a complete understanding of presenteeism, one probably needs to take into consideration all the different approaches and insights from various disciplines.

From an economic point of view, presenteeism is inefficient, being stressful for workers and costly to firms, and resulting in a suboptimal allocation of labour. Both workers and firms would be better off if the extent of presenteeism could be reduced, though this proves difficult in practice. Our results imply that presenteeism might be lower if workers could credibly reveal their health status to employers, but it is unclear how this could be accomplished as long as sickness cannot be measured objectively. In addition, presenteeism (and absenteeism) can be influenced to a certain degree by firms' setting of wages and devising appropriate organisational practices and by changes in the institutional framework concerning sick pay, although the latter has proved not to be politically feasible in Germany (see, e.g., Puhani and Sonderhof, 2010). Finally, our theoretical and empirical results point to the relevance of dismissal protection for engaging in presenteeism. Yet, given the small effect found in our study and the well-known labour 
market effects of dismissal protection legislation we would not recommend increasing the extent of dismissal protection just to combat presenteeism. More specific and better founded recommendations might only be possible if we had employer-employee panel data on presenteeism covering several years and relevant changes in the institutional framework and in firms' personnel policies. Unfortunately, no such data are currently in sight.

\section{References}

Angrist, J.D. and Pischke, J.S. (2009), Mostly Harmless Econometrics. An Empiricist's Companion, Princeton, NJ: Princeton University Press.

Arnold, D. (2015), 'Determinants of the annual duration of sickness presenteeism: Empirical evidence from European data,' Labour, forthcoming.

Aronsson, G. and Gustafsson, K. (2005), 'Sickness presenteeism: Prevalence, attendance-pressure factors, and an outline of a model for research,' Journal of Occupational and Environmental Medicine, 47(9):958-965.

Aronsson, G., Gustafsson, K., and Dallner, M. (2000), 'Sick but yet at work. an empirical study if sickness presenteeism,' Journal of Epidemiology and Community Health, 54(7):502-509.

Barmby, T. and Larguem, M. (2009), 'Coughs and sneezes spread diseases: An empirical study on absenteeism and infectious illness,' Journal of Health Economics, 28(5):1012-1017.

Barmby, T., Sessions, J.G., and Treble, J.G. (1994), 'Absenteeism, efficiency wages and shirking,' Scandinavian Journal of Economics, 96(4):561-566.

Beemsterboer, W., Stewart, R., Groothoff, J., and Nijhuis, F. (2009), 'A literature review on sick leave determinants (1984-2004),' International Journal of Occupational Medicine and Environmental Health, 22(2):169-179.

Bierla, I., Huver, B., and Richard, S. (2013), 'New evidences on absenteeism and presenteeism behaviour,' International Journal of Human Resource Management, 24(7):1536-1550.

Böckerman, P. and LaukKanen, E. (2009), 'What makes you work while you are sick? Evidence from a survey of workers,' European Journal of Public Health, 20(1):43-46.

Brown, S. and Sessions, J.G. (2004), 'Absenteeism, 'presenteeism', and shirking,' Economic Issues, 9(1):15-21.

ChatterJi, M. and Tilley, C.L. (2002), 'Sickness, absenteeism, presenteeism, and sick pay,' Oxford Economic Papers, 54(4):669-687. 
Coles, M.G. and Treble, J.G. (1993), 'The price of worker reliability,' Economic Letters, 41(2):149-155.

DE VRoome, E. (2006), Prevalence of sickness absence and 'presenteeism', URL: http://eurofound.europa.eu/ewco/2006/07/NL0607019I.htm, retrieved at 27/08/2014.

ElWert, F. and Winship, C. (2014), 'Endogenous selection bias: The problem of conditioning on a collider variable,' Annual Review of Sociology, 40(1):31-53.

Eurofound (2012), Fifth European Working Conditions Survey, Luxembourg: Publications Office of the European Union.

Gosselin, E., Lemyre, L., and Corneil, W. (2013), 'Presenteeism and absenteeism: Differentiated understanding of related phenomena,' Journal of Occupational Health Psychology, 18(1):75-86.

Hall, A., Siefer, A., and Tiemann, M. (2014), BIBB/BAuA Employment Survey of the Working Population on Qualification and Working Conditions in Germany 2012. suf_3.0, Research Data Center at BIBB (ed.), GESIS Cologne (data access), Bonn: Federal Institute for Vocational Education and Training.

Hansen, C.B. and Andersen, J.H. (2008), 'Going ill to work - what personal circumstances, attitudes and work-related factors are associated with sickness presenteeism?' Social Science and Medicine, 67(6):956-964.

(2009), 'Sick at work - a risk factor for long-term sickness absence at a later date?' Journal of Epidemiology and Community Health, 63(5):397-402.

Hemp, P. (2004), 'Presenteeism: At work - but out of it,' Harvard Business Review, 82(10):49-58.

Johansen, V., Aronsson, G., and Marklund, S. (2014), 'Positive and negative reasons for sickness presenteeism in Norway and Sweden: A cross-sectional survey,' BMJ Open, 4(2):1-6.

Johns, G. (2010), 'Presenteeism in the workplace: A review and research agenda,' Journal of Organizational Behavior, 31(4):519-542.

Oldenburg, C. (2012), 'Präsentismus - die zweite Seite der Gesundheitsmünze,' in A. Lohmann-Haislah (ed.), 'Stressreport Deutschland 2012: Psychische Anforderungen, Ressourcen und Befinden,' pp. 134-142, Dortmund et al.: Bundesanstalt für Arbeitsschutz und Arbeitsmedizin.

Pedersen, K.M. and Skagen, K. (2014), The economics of presenteeism: A discrete choice and count model framework, Centre of Health Economics Research, University of Southern Denmark, COHERE Working Paper No. 2014:2.

Pichler, S. and Ziebarth, N.R. (2015), The pros and cons of sick pay schemes: A method to test for contagious presenteeism and shirking behavior, Institute for the Study of Labor, Bonn, IZA Discussion Paper No. 8850.

Preisendörfer, P. (2010), 'Präsentismus, Prävalenz und Bestimmungsfaktoren unterlassener Krankmeldungen bei der Arbeit,' Zeitschrift für Personalforschung, 24(4):401-408. 
Puhani, P.A. and Sonderhof, K. (2010), 'The effects of a sick pay reform on absence and on health-related outcomes,' Journal of Health Economics, 29(2):285-302.

Rohrbach-Schmidt, D. and Hall, A. (2013), BIBB/BAuA Employment Survey 2012, Federal Institute for Vocational Education and Training, Bonn, BIBB-FDZ Data and Methodological Reports No. 1/2013.

Schultz, A.B. and Edington, D.W. (2007), 'Employee health and presenteeism: A systematic review,' Journal of Occupational Rehabilitation, 17(3):547-579.

Shapiro, C. and Stiglitz, J.E. (1984), 'Equilibrium unemployment as a worker discipline device,' American Economic Review, 74(3):433-444.

SkÅTun, J.D. (2003), 'Take some days off, why don't you? Endogenous sick leave and pay,' Journal of Health Economics, 22(3):379-402.

Steinke, M. and BadurA, B. (2011), Präsentismus: Ein Review zum Stand der Forschung, Dortmund et al.: Bundesanstalt für Arbeitsschutz und Arbeitsmedizin.

Tiemann, M., Schade, H.J., Helmrich, R., Hall, A., Braun, U., and Bott, P. (2008), Berufsfeld-Definitionen des BIBB auf Basis der Klassifikation der Berufe 1992, Bundesinstitut für Berufsbildung, Bonn, Wissenschaftliche Diskussionspapiere Nr. 105.

Treble, J. and Barmby, T. (2011), Worker Absenteeism and Sick Pay, Cambridge: Cambridge University Press.

Vogt, J., Badura, B., and Hollmann, D. (2009), 'Krank bei der Arbeit: Präsentismusphänomene,' in J. Böcken, B. Braun, and J. Landmann (eds.), 'Gesundheitsmonitor 2009: Gesundheitsversorgung und Gestaltungsoptionen aus der Perspektive der Bevölkerung,' pp. 179-202, Gütersloh: Verlag Bertelsmann Stiftung.

Weiss, A. (1985), 'Absenteeism and wages,' Economics Letters, 19(3):277-279.

Wooldridge, J.M. (2010), Econometric Analysis of Cross Section and Panel Data, Cambridge, MA: MIT Press, 2nd edn. 